\title{
Design and Fabrication of Quartz MEMS-based Monolithic Vibrating Beam Accelerometer
}

\author{
Jinxing Liang, Qi Tang, and Shengshou Lin \\ Key Laboratory of Micro-Inertial Instrument and Advanced Navigation Technology, Ministry of Education, \\ School of Instrument Science and Engineering, Southeast University, Nanjing 210096, China
}

(Received September 24, 2020; accepted January 11, 2021; online published January 19, 2021)

Keywords: quartz, vibrating beam accelerometer, monolithic, high sensitivity

We present a novel monolithic quartz vibrating beam accelerometer (QVBA), which is mainly composed of a single-beam resonator, a base-proof mass structure, and a vibration isolation frame. The sensor measures the acceleration signal perpendicular to the proof mass surface, which is transferred into an inertial force through the proof mass. We attempted to improve the sensing structure configuration to enhance the sensitivity and suppress cross-axis sensitivity. A two-step wet etching process was successfully developed to fabricate the designed sensor, and a simple self-excitation oscillation circuit was designed to drive the fabricated accelerometer. The static measurement result demonstrated high sensitivity of about $54.46 \mathrm{~Hz} / \mathrm{g}$, and the bias stability was evaluated to be $33 \mu g(1 \sigma)$. In the temperature range from -40 to $60{ }^{\circ} \mathrm{C}$, the bias frequency shift was below $1.9 \mathrm{ppm} /{ }^{\circ} \mathrm{C}$.

\section{Introduction}

A double-ended vibrating beam resonator is a well-known force transducer, which usually vibrates in the flexural mode when sensing a longitudinal force. A vibrating beam resonator can directly work as a force gauge and measure pressure or acceleration by incorporating an elastic diaphragm or second-order spring-mass system. ${ }^{(1)}$ This kind of sensor has attracted continuous attention owing to its digital output, large measurement range, and good dynamic characteristics. Benefiting from mature microfabrication techniques, silicon-based MEMS resonant accelerometers have been widely studied and reported. ${ }^{(2-6)}$ Compared with the commonly used silicon, crystal quartz has a higher quality factor and excellent temperature frequency stability, which are very important for resonant sensors. Furthermore, the vibration of a quartz vibrating beam resonator can be easily excited by utilizing the piezoelectricity of the material without the need for an additional excitation structure, as in the case of using silicon.

Most silicon-based monolithic resonant accelerometers utilize a double-ended tuning fork (DETF) as the force-frequency transducer, which is well known to easily maintain high-quality vibration due to the symmetrical dual-beam structure. Unfortunately, in the case of using quartz, which is usually micromachined by anisotropic wet etching, the cross section of the

*Corresponding author: e-mail: j-liang@seu.edu.cn

https://doi.org/10.18494/SAM.2021.3004 
beam has a very complex asymmetric profile. That is why DETF-based quartz accelerometers usually adopt separate bodies and are manufactured post-assembly. A DETF resonator is fabricated using a thin wafer substrate, and the proof mass structure is made of a metal, ${ }^{(7)}$ silicon, ${ }^{(8)}$ or a quartz wafer. ${ }^{(9)}$ The mismatch of the coefficient of thermal expansion among the quartz, metal, and bonding material is expected to lead to a long-time reliability problem. On the other hand, a single-beam resonator is another commonly used force transducer, which in principle has twice the force sensitivity of a double-beam resonator and no strict requirement on the cross-sectional profile of the beam. This means that it is possible to design a monolithic sensor structure incorporating an on-wafer vibration isolation platform. ${ }^{(10,11)}$ Recently, a singlebeam-based monolithic quartz accelerometer has been successfully developed by ONERA (France), in which an external anti-vibration isolation structure is inserted that prevents vibration energy leaking into the mounting part. ${ }^{(12-15)}$

Generally, the design of a high-precision resonant accelerometer should address several issues. Firstly, the sensor should be designed for high stability. This means that the parent material of the accelerometer should be highly stable, such as chemically inert to the environment, and have low power consumption and excellent temperature characteristics. Secondly, the sensor should have long-term reliability, for which a simple sensor configuration without the assembly of joint parts is preferable wherever possible. Finally, the accelerometer should be sensitive to acceleration along the sensing axis and insensitive to acceleration in all other directions.

To meet these requirements, we designed a novel quartz monolithic accelerometer with an improved structure, the sensing element of which is composed of a single-beam force transducer, proof mass, and support. The proof mass and support are linked by a pair of hinges, which are much thinner than the proof mass and have the same thickness-center plane as the proof mass. The proposed sensing structure has the advantages of improved measurement sensitivity and greatly suppressed cross-axis disturbance compared with the reported structure (ONERA). Detailed information will be given in the following design section.

\section{Working Principle and Design Method}

The uniaxial vibrating beam accelerometer is schematically illustrated in Fig. 1 and is composed of a monolithic crystalline Z-cut quartz substrate. The proof mass is connected to the support by two hinges that permit the proof mass to rotate with respect to the support about the hinge axis. One end of the single vibrating beam of the force transducer is connected to the support and the other end is connected to the proof mass. Both the vibrating beam and the two hinges have a thickness substantially less than that of the support and proof mass.

\subsection{General operating principle}

The sensing direction is perpendicular to the proof mass plane, and the proof mass is much wider than the vibrating beam and flexures. When an external acceleration is applied to the proof mass along the sensing axis, the produced inertial force is magnified and coupled onto 


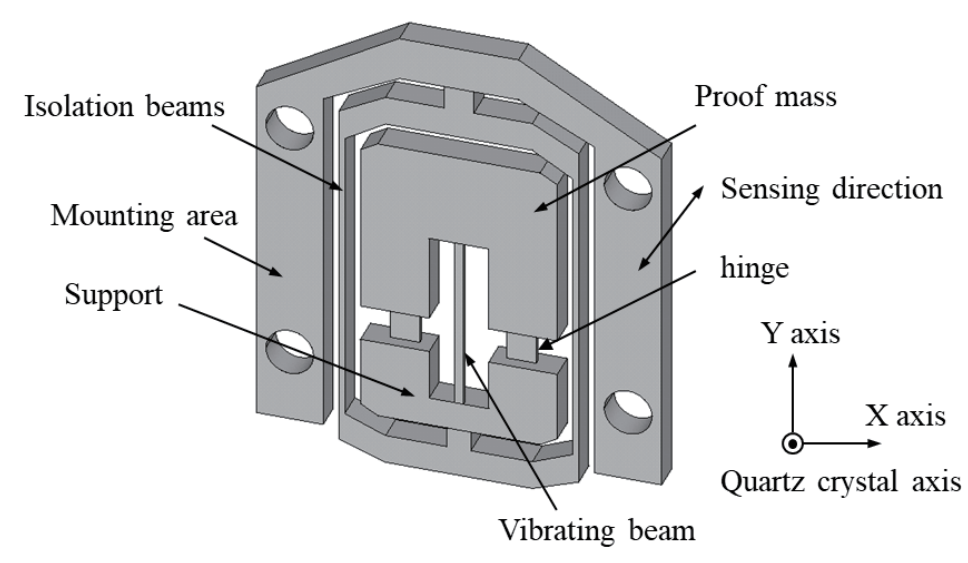

Fig. 1. Structure of uniaxial vibrating beam accelerometer.

the single beam through a leverage mechanism. The single vibrating beam works in an in-plane flexional mode, the resonating frequency of which increases upon a tension force and decreases upon a compression force along the longitudinal direction. The beam length is aligned along the quartz crystallographic $Y$ axis, and the beam width is aligned along the crystallographic $X$ axis. Taking advantage of the inverse piezoelectric effect of quartz, the flexional mode vibration can be easily excited even by only surface electrodes through the piezoelectric coefficient $e_{12}$, where the detailed electrode pattern design has been reported in our previous publication. ${ }^{(16)}$ The key sensing element includes the proof mass, flexures, and vibrating beam resonator, which determine the sensitivity, measurement range, and so on. In addition to the sensing elements, an isolation frame is implemented between the sensing element and the mounting area. The isolation frame and the sensing element constitute a second-order isolator resonator system, in which the isolation frame serves as a spring element and the sensing element acts as a proof mass. The isolator works as a passive mechanical low-pass-filter resonator, which is usually used for attenuating high-frequency environment vibrations. ${ }^{(11)}$ On the other hand, the isolator can also attenuate high-frequency single-beam vibration, which prevents the energy from leaking into the mounting area and maintains the resonance of the vibrating beam with a high $\mathrm{Q}$ value.

\subsection{Design and modeling considerations}

\subsubsection{Vibrating beam force transducer}

The nominal resonant frequency $f_{0}$ of a single vibrating beam in flexional mode is given by

$$
\begin{gathered}
f_{0}=\frac{a^{2}}{2 \pi l^{2}} \sqrt{\frac{E I}{\rho A}}, \\
a=\frac{1+2 n}{2} \cdot \pi,
\end{gathered}
$$


wherein $a$ is determined by the vibration mode number $n$ as in Eq. (2), $l$ is the length of the vibrating beam, $E=7.8 \times 10^{10} \mathrm{~Pa}$ is the Young's modulus of the quartz material, $I$ is the moment of inertia of the area, $A$ is the cross-sectional area of the beam, and $\rho=2650 \mathrm{~kg} / \mathrm{m}^{3}$ is the density of quartz.

When a longitudinal axial force $F$ is applied, the resonating frequency of the vibrating beam will become

$$
f=f_{0} \sqrt{1+\frac{1}{a^{3}} \cdot \tanh \frac{a}{2} \cdot\left(a \tanh \frac{a}{2}-2\right) \cdot \frac{l^{2}}{E I} \cdot F} .
$$

Generally, the fundamental vibration mode is used as a force transducer, namely, $n=1(a=4.73)$. Then Eqs. (1) and (3) can be rewritten as

$$
\begin{gathered}
f_{0}=\frac{22.4}{2 \pi l^{2}} \sqrt{\frac{E I}{\rho A}}, \\
f=f_{0} \sqrt{1+0.0246 \cdot \frac{l^{2}}{E I} \cdot F} .
\end{gathered}
$$

Furthermore, for an ideal beam with a rectangular cross section, the moment of inertia of the area, $I$, and the cross-section area $A$ can be calculated as

$$
\begin{aligned}
& I=\frac{w^{3} t_{1}}{12}, \\
& A=w t_{1},
\end{aligned}
$$

where $w$ is the beam width in the vibration direction and $t_{1}$ is the beam thickness. The fundamental resonating frequency of the vibrating beam can be simplified to

$$
f_{0}=\frac{22.4 w}{2 \pi l^{2}} \sqrt{\frac{E}{\rho}}
$$

It can be clearly seen that the resonating frequency of the beam is mainly determined by the beam width and length. For a vibrating beam with a fixed nominal frequency, the narrower the beam width, the higher the force-frequency sensitivity. However, when designing the beam structure, fabrication issues should be considered. A too narrow beam width will make it difficult to arrange the excitation electrode pattern.

\subsubsection{Transduction mechanisms of the inertial force}

Figure 2(a) shows a schematic diagram of the key sensing element of the proposed accelerometer structure and Fig. 2(b) shows the ONERA structure. An obvious difference 


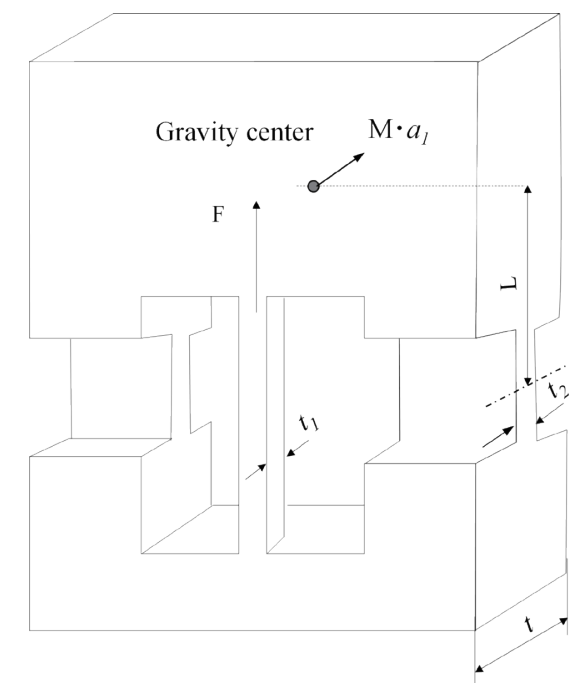

(a)

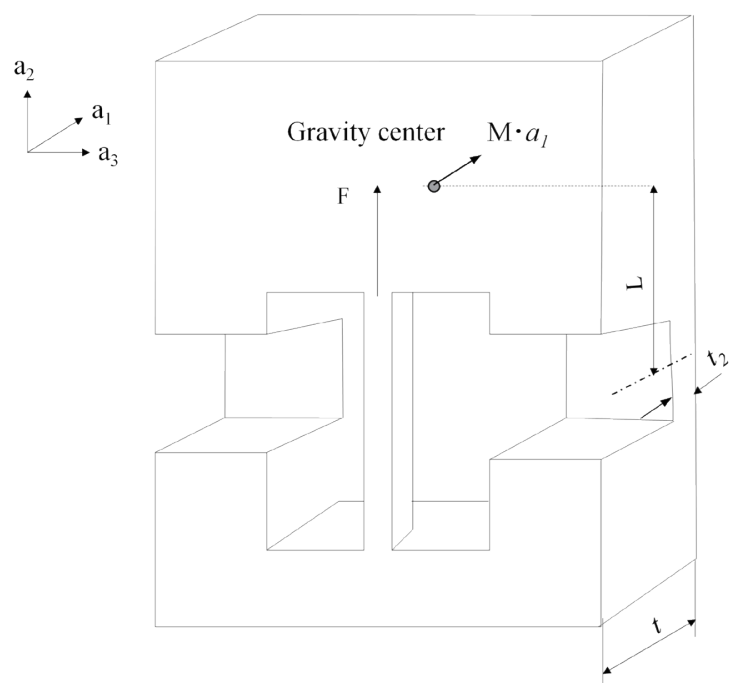

(b)

Fig. 2. Schematic diagrams of the sensing element structures. (a) Proposed structure. (b) ONERA structure.

between the two structures is the hinge position. In our proposed structure, the hinge is located at the center of the wafer thickness, whereas it is on one side in the ONERA structure. In response to acceleration, the proof mass rotates around the hinge in response to the produced inertial force. The inertial force is magnified and transferred onto the vibrating beam via a leverage principle. For our proposed structure [Fig. 2(a)], the force $F_{1}$ exerted on the vibrating beam along the longitudinal direction to induce acceleration $a_{1}$ in the sensing direction can be expressed by Eq. (9), neglecting the small rotation angle. ${ }^{(13)}$

$$
F_{1 a}=\frac{2 M \cdot a_{1} \cdot L}{t-t_{1}}
$$

Here, $M$ is the total proof mass, $t$ and $t_{1}$ are the wafer thickness and vibrating beam thickness, respectively, and $L$ is the distance between the center of gravity of the proof mass and the hinge center. The inertial force is magnified by a factor of $2 L /\left(t-t_{1}\right)$, where a high value of the inertial force is preferable for high sensitivity.

For the ONERA structure, the force $F_{1}$ exerted upon the same acceleration $a_{1}$ can be expressed by

$$
F_{1 b}=\frac{2 M \cdot a_{1} \cdot L}{2 t-t_{1}-t_{2}} .
$$

Here, $t_{2}$ is the hinge beam thickness. We perform a simple calculation by neglecting the beam thickness $\left(t_{1}\right)$ and hinge thickness $\left(t_{2}\right)$, which are much smaller than the wafer thickness $(t)$. For the same applied acceleration $a_{1}$, the produced axial force is $F_{1 b}=M \cdot a_{1} L / t$ for the ONERA structure [Fig. 2(b)] and $F_{1 a}=2 M \cdot a_{1} L / t$ for our proposed structure [Fig. 2(a)]. Namely, the sensitivity can potentially be improved twofold. Accurately, taking into account the thickness of the beam and hinge, the increase in the sensitivity will be slightly smaller than twofold. 
Regarding the cross-axis acceleration $a_{2}$, the exerted force $F_{2}$ is zero for our proposed structure [Fig. 2(a)] because the inertial force $M \cdot a_{2}$ is parallel to the longitudinal direction of the beam. The produced inertial force is mainly withstood by the flexures because it is acting in the center plane of the flexures. However, for the ONERA structure, half of the produced inertial force will be applied on the sensing beam resonator. This means that our proposed structure can greatly suppress this directional crosstalk.

The acceleration $a_{3}$ acts in the middle plane of the proof mass and is perpendicular to the longitudinal direction of the beam, so in principle applies no tensile or compression force to the vibrating beam resonator. As a result, there are no frequency shifts caused by crosstalk from the two directions perpendicular to the sensing axis. That means that the proposed sensor configuration could be highly insensitive to cross-axis disturbances.

\subsubsection{Vibration isolation between vibrating beam and mounting part}

When a single-beam resonator is used as a force frequency transducer, a vibration isolator is necessary to maintain a high-quality resonation. ${ }^{(17-19)}$ Depending on the incorporation mechanism, there are two ways to design the isolator system. The first way is to directly arrange a pair of beam-mass structures at the two ends of the working vibrating beam. The isolator is used to counterbalance the vibrational force and torque produced at the root of the beam ends. ${ }^{(1,18)}$ This configuration incorporates the isolator inside the support-proof mass structure. However, this greatly increases the size of the sensor because the isolator is installed in the longitudinal direction of the working beam. The second way is to design the isolator system outside the support-proof mass structure, and the isolator beam works as a low-pass filter, which has a resonant frequency much lower than that of the working beam. ${ }^{(13)}$ We adopt the second method, which is also used in the ONERA structure. ${ }^{(13)}$ In this configuration, the isolator attenuates the high-frequency vibration of the working beam, which prevents the vibration energy from leaking into the outer mounting part. In fact, it can also prevent the environmental in-plane vibration from coupling into the working beam. The measurement range is designed to be $\pm 50 \mathrm{~g}$ in this research. Table 1 shows the key dimensions of the designed monolithic accelerometer.

Figure 3 shows the main vibration modes simulated by finite element analysis using the commercial software ANSYS with the parameters shown in Table 1. Figure 3(a) shows the proof

Table 1

Physical dimensions of the designed accelerometer.

\begin{tabular}{lcc}
\hline Property & Variable & Value $(\mu \mathrm{m})$ \\
\hline \multirow{3}{*}{ Vibrating beam } & Length & 2900 \\
& Width & 70 \\
& Thickness & 35 \\
\hline \multirow{3}{*}{ Proof mass } & Length & 2900 \\
& Width & 3300 \\
& Thickness & 300 \\
\hline \multirow{3}{*}{ Hinge } & Length & 1000 \\
& Width & 500 \\
& Thickness & 30 \\
\hline
\end{tabular}




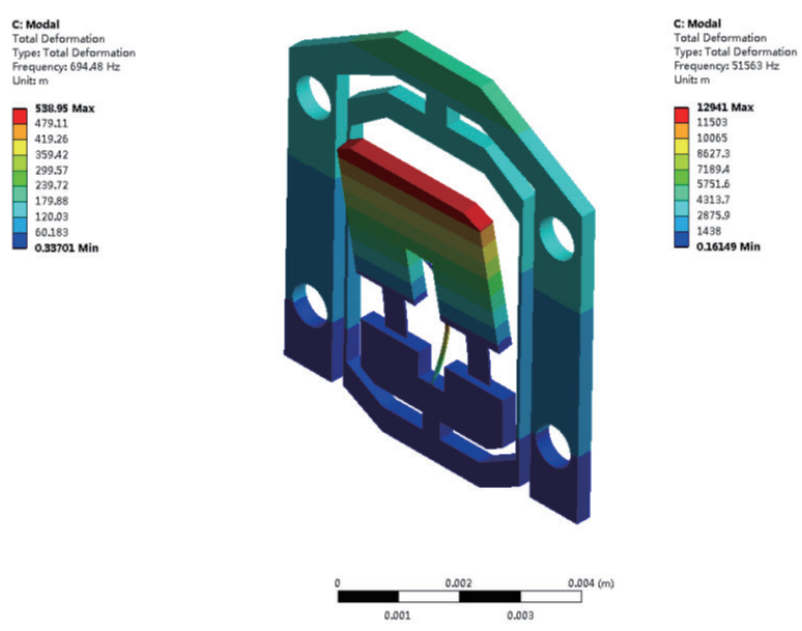

(a)

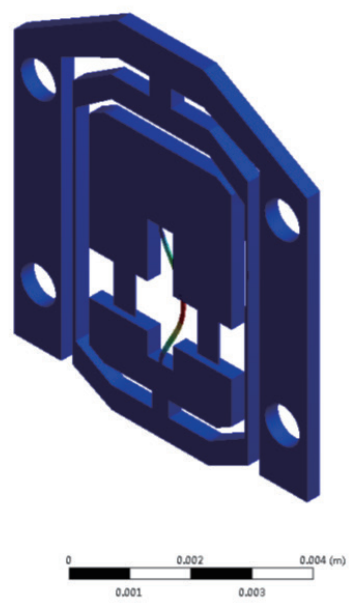

(b)

Fig. 3. (Color online) Simulated main vibration modes: (a) proof mass vibration mode; (b) vibrating beam in-plane flexional mode.

mass vibration mode, which is an important parameter determining the dynamic range of the measurement. The simulated natural frequency of the proof mass is $694 \mathrm{~Hz}$. The frequency of the measured acceleration signal should be much lower than the proof mass frequency; otherwise, a sensitivity error will be produced with increasing actual vibration frequency. Figure 3(b) shows the in-plane flexion vibration mode of the resonating beam. The simulated resonating frequency is $51563 \mathrm{~Hz}$.

\section{Fabrication, Assembly, and Evaluation}

\subsection{Fabrication process}

A two-step quartz etching process based on our well-established wet etching process is developed to form the hinge-centered structure. ${ }^{(20)}$ The fabrication of the designed accelerometer starts from a $300-\mu \mathrm{m}$-thick single-crystal quartz wafer.

Figure 4 shows the detailed fabrication process of the accelerometer. The process is as follows: (1) Wash the quartz wafer using piranha solution $\left(\mathrm{H}_{2} \mathrm{SO}_{4}: \mathrm{H}_{2} \mathrm{O}_{2}=3: 1\right)$ with a temperature of $110{ }^{\circ} \mathrm{C}$ for $15 \mathrm{~min}$, then sputter chromium $(\mathrm{Cr}, 20 \mathrm{~nm})$ and gold $(\mathrm{Au}, 100 \mathrm{~nm})$ bilayer metal films on both sides. The bilayer $\mathrm{Au} / \mathrm{Cr}$ metal films are used to protect the quartz from etching. After etching the quartz, some $\mathrm{Au} / \mathrm{Cr}$ patterns in specific areas are left to serve as excitation electrodes, bonding pads, and leading wires. (2) Spin-coat photoresist (S1808) on both sides of the quartz wafer, and then pattern the lower surface. This step defines the pattern to be first etched. (3) Etch the $\mathrm{Au} / \mathrm{Cr}$ films and remove the photoresist, then recoat and pattern the quartz wafer on both sides. This step defines the area to be etched in the second step of etching, in which etching occurs from both sides. Before etching, the quartz wafer should be hardbaked for $30 \mathrm{~min}$ at $140{ }^{\circ} \mathrm{C}$ to ensure resistance to the aggressive quartz etchant. (4) Etch the 


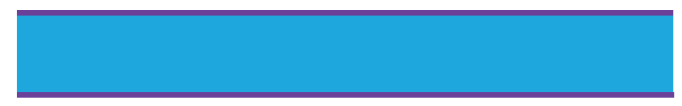

(1) Washing quartz wafer and sputtering $\mathrm{Au} / \mathrm{Cr}$ films

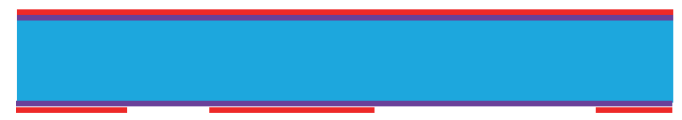

(2) Spin-coating photoresist and patterning

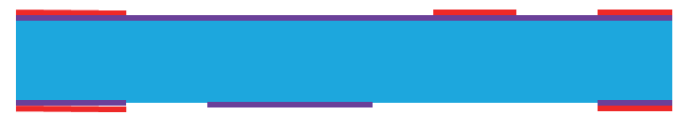

(3) Etching $\mathrm{Au} / \mathrm{Cr}$ and re-patterning photoresist

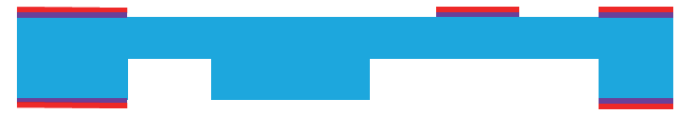

(4) Etching quartz, followed by $\mathrm{Au} / \mathrm{Cr}$ etching

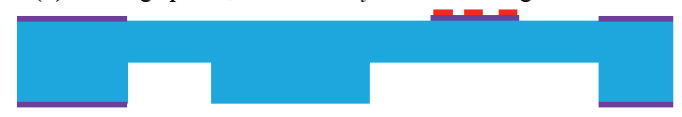

(5) Patterning excitation electrode

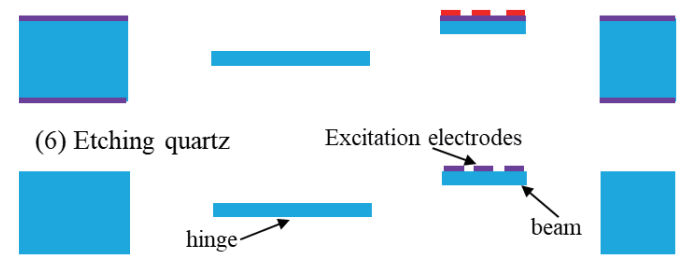

(7) Etching $\mathrm{Au} / \mathrm{Cr}$ and removing photoresist

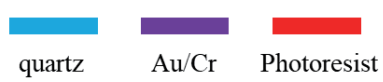

Fig. 4. (Color online) Fabrication process flow of accelerometer structure.

quartz in saturated ammonium bifluoride solution at $85{ }^{\circ} \mathrm{C}$ to a depth of $130 \mu \mathrm{m}$, followed by $\mathrm{Au} / \mathrm{Cr}$ etching and photoresist removal. (5) Spin-coat and pattern the quartz wafer on the upper surface. This step defines the pattern of the excitation electrodes, bonding pads, and leading wires. (6) Etch the quartz on both sides to a depth of $135 \mu \mathrm{m}$ on each side. Finally, wash the quartz wafer with piranha solution $\left(\mathrm{H}_{2} \mathrm{SO}_{4}: \mathrm{H}_{2} \mathrm{O}_{2}=3: 1\right)$ for $15 \mathrm{~min}$.

\subsection{Assembly and evaluation}

After fabrication, the monolithic sensor chips are cut from the quartz wafer. Vacuum packaging is needed to improve the $\mathrm{Q}$ value of the accelerometer. Common package materials include plastics, ceramics, and metals, and a ceramic package is selected in this study because the coefficient of thermal expansion of the ceramic is close to that of quartz. The quartz structure is mounted into a ceramic package (by gluing), and quartz gaskets are used to guarantee the vibration of the active part of the sensitive elements. The excitation electrode pads are wire-bonded to the ceramic package pads. The sensitive elements operate under vacuum, and the characteristics ( $Q$ value, resonant frequency, equivalent circuit parameters) 
are measured using an Agilent 4294A impedance analyzer. On the basis of the measured circuit parameters, a multiple common-emitter series oscillator circuit with negative feedback is designed and fabricated to drive the sensitive elements.

The oscillator circuit is shown in Fig. 5 and includes a frequency-selecting network, a twostage common-emitter amplifier, a phase-shifted circuit, a low-pass filter circuit, and a circuit for adjusting the output voltage amplitude. The quartz vibrating beam accelerometer (QVBA) frequency-selecting network is connected to the two-stage common-emitter amplifier and the phase-shifted circuit to serve as a closed-loop oscillator. The output of the common-emitter amplifier and the phase-shifted circuit is connected to the input of the filter circuit to filter the high-frequency noise. After adjusting the voltage amplitude, the output signal is connected to a digital circuit.

\section{Results and Discussion}

Figure 6 shows the fabricated and bonded sensor in a ceramic package, where the dimensions of the quartz chip and ceramic package are $6.8 \times 8$ and $14 \times 14 \mathrm{~mm}^{2}$, respectively.

After vacuum sealing, the vibration characteristics of the sensor are measured. Figure 7(a) shows the experimental setup for measuring characteristics using an Agilent 4294A impedance analyzer. The sensitive element is mounted into a ceramic package and the package sensor is fixed on a precise goniometer stage. Figure 7(b) shows the resonating characteristics of the package sensor. The Q value is around 8000 and the resonant frequency is $51.58 \mathrm{kHz}$.

As mentioned above, an oscillation circuit based on the measured equivalent circuit parameters is designed and fabricated. The packaged sensor is bonded on the circuit board then assembled into an aluminum alloy shell. Figure 8(a) shows the bonded sensor on the printed circuit board. Figure 8(b) shows the experimental setup for measuring the static characteristic of the tested accelerometer. A digital regulated DC power supply provides a voltage of $8-10 \mathrm{~V}$

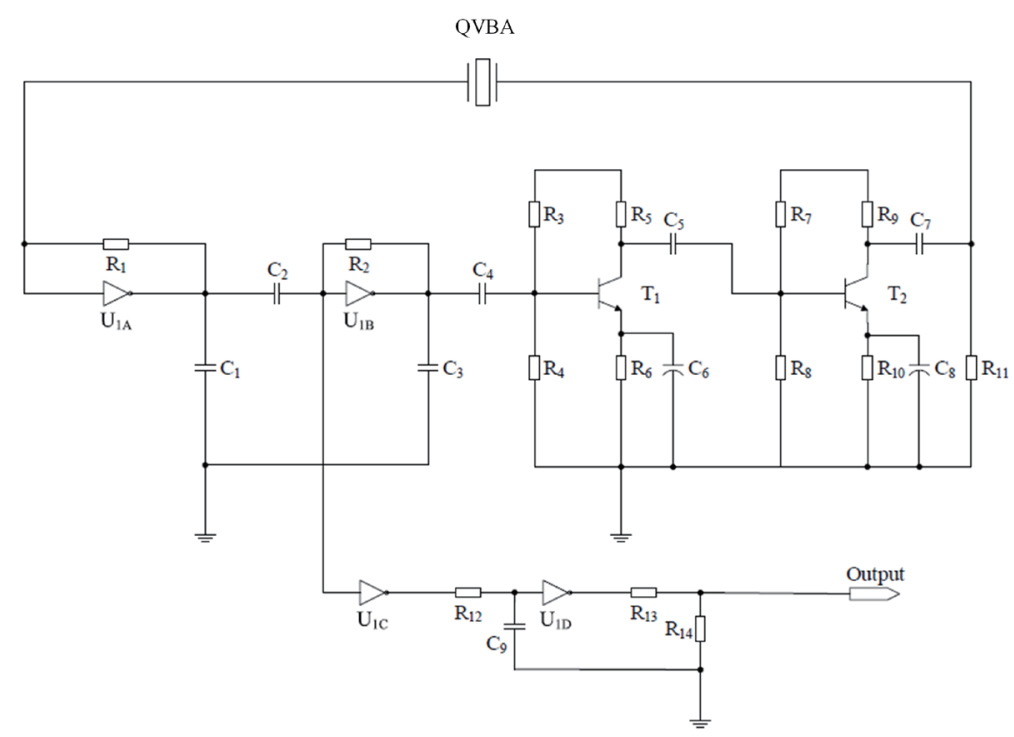

Fig. 5. Oscillator circuit of QVBA. 


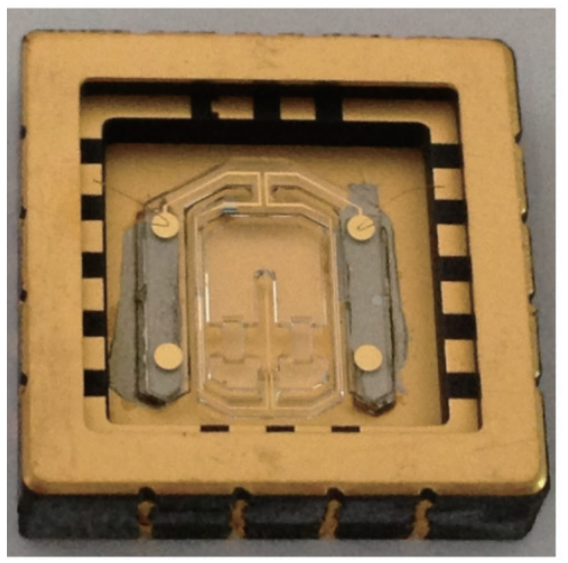

(a)

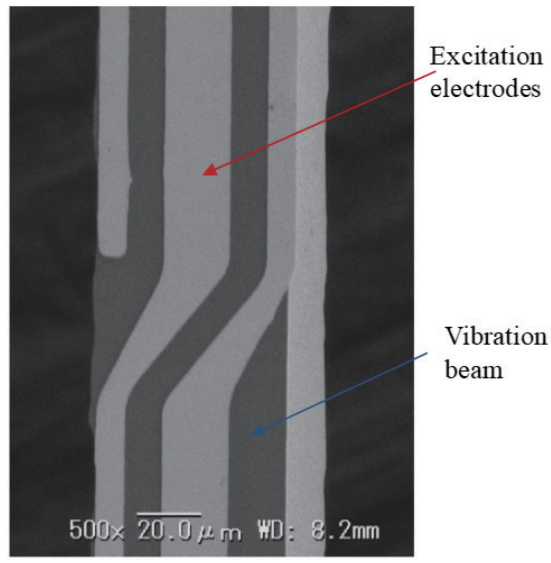

(b)

Fig. 6. (Color online) Images of the fabricated and bonded sensor chip: (a) chip bonded in the ceramic package; (b) close-up SEM image of the beam and excitation electrodes.

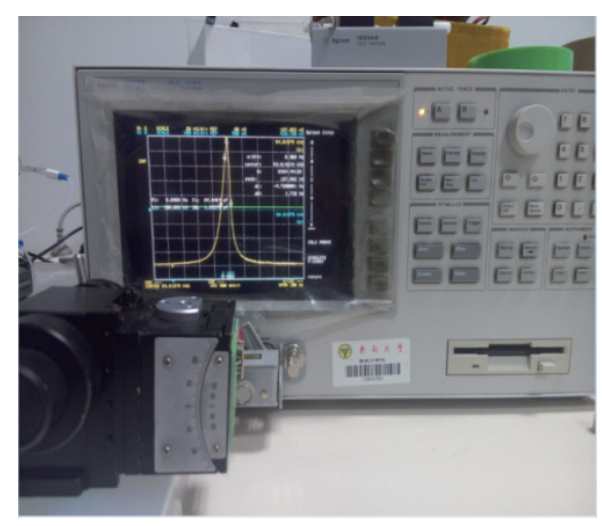

(a)

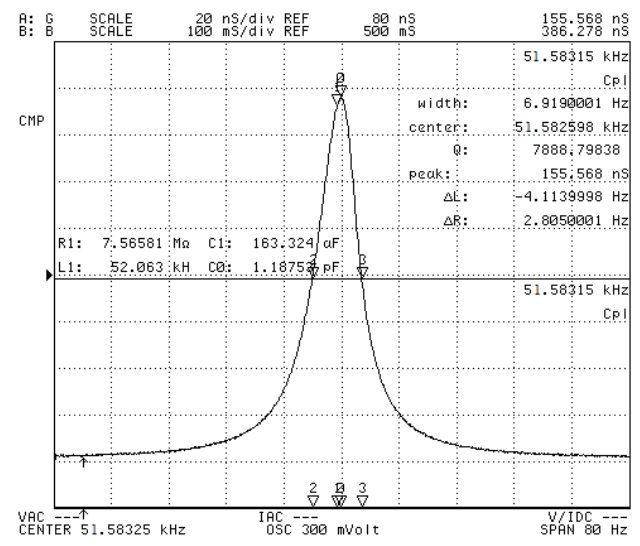

(b)

Fig. 7. (Color online) Measurement of QVBA: (a) experimental setup; (b) measured vibration characteristics.

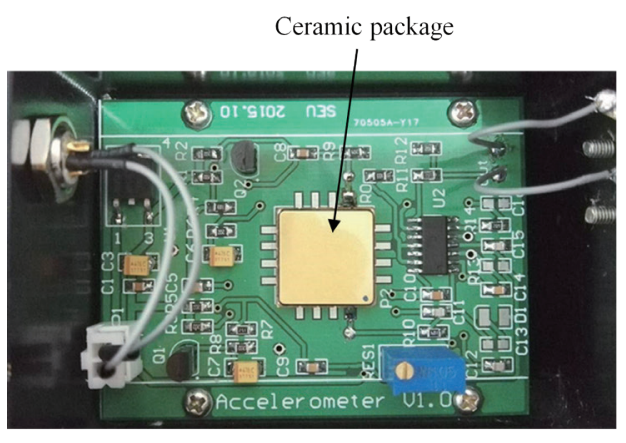

(a)

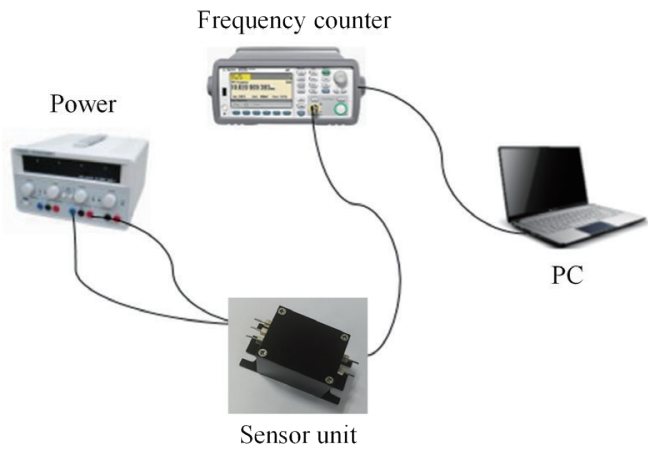

(b)

Fig. 8. (Color online) Images of assembled sensor: (a) mounting on oscillation circuit; (b) setup of experimental measurement. 
for the accelerometer, and the output frequency is obtained using an Agilent 53220 frequency counter with a sampling time of $1 \mathrm{~s}$. The frequency counter is connected to a computer by GPIB wires to record and display frequency curves in real time. A precision angle dividing head $(0.25$ arcsecond) that can produce static acceleration in the range from $-1 g$ to $+1 g$ is used to evaluate the sensor performance.

First, the resonant frequencies upon $+1 g$ and $-1 g$ acceleration are measured by rotating the angle dividing head. The sensing scale factor is measured by taking the resonant frequency difference upon $+1 g$ and $-1 g$ acceleration, and the scale factor stability and scale factor are measured. The scale factor is calculated to be $54.46 \mathrm{~Hz} / \mathrm{g}$ by dividing the frequency difference by 2 . The scale factor stability and repeatability are measured to be 86 and $23 \mathrm{ppm}$, respectively. The detailed measurement conditions and data calculation method can be found in a previous report. ${ }^{(3)}$

Figure 9 shows the bias stability of the tested accelerometer for $1 \mathrm{~h}$ after a 30 min electrical warm up. The calculated standard deviation $(1 \sigma)$ is $33 \mu g$.

The bias stability as a function of temperature is measured by placing the sensor (including the oscillation circuit) in an oven. The oven temperature is shifted in a cycle $(20,40,60,40,20$, $0,-20,-40,-20,0,20^{\circ} \mathrm{C}$ ), and the oven temperature is stabilized for $1 \mathrm{~h}$ at each temperature. Figure 10 shows the resonant frequency shift during the temperature cycle. The maximum frequency shift in the range of -40 to $60{ }^{\circ} \mathrm{C}$ is below $10 \mathrm{~Hz}$, which corresponds to $1.9 \mathrm{ppm} /{ }^{\circ} \mathrm{C}$. This result clearly demonstrates the excellent frequency stability of the quartz.

As a result of the improved sensing structure, high sensitivity was achieved. The improved measurement sensitivity and the suppression of the cross-axis response are next demonstrated by simulation. Figure 11 separately shows the simulated resonant frequency upon applying acceleration based on the sensor dimensions in Table 1 from the measurement $Z$ direction, cross-axis $Y$ direction, and cross-axis $X$ direction.

It can be clearly seen from Fig. 11(a) that the measurement sensitivity is increased 1.8-fold for the proposed sensor structure. Also, the cross-axis talk is greatly suppressed compared with the ONERA structure [Figs. 11(b) and 11(c)]. Furthermore, the developed two-step quartz etching process gives the design freedom, and the beam thickness and hinge thickness can be designed with different values, which could not be realized with the conventional one-step etching process. ${ }^{(14)}$

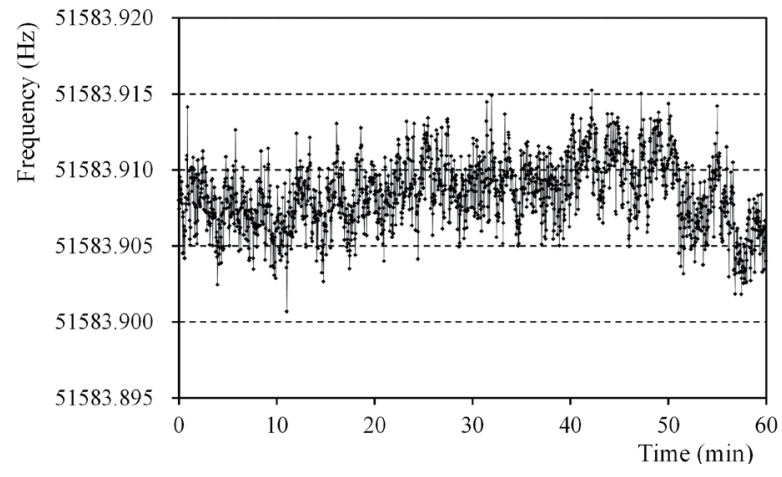

Fig. 9. Measured bias stability over $1 \mathrm{~h}$.

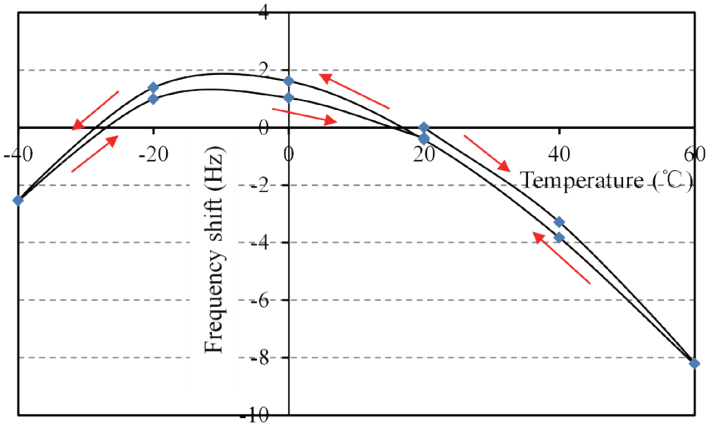

Fig. 10. (Color online) Dependence of resonant frequency shift on temperature. 


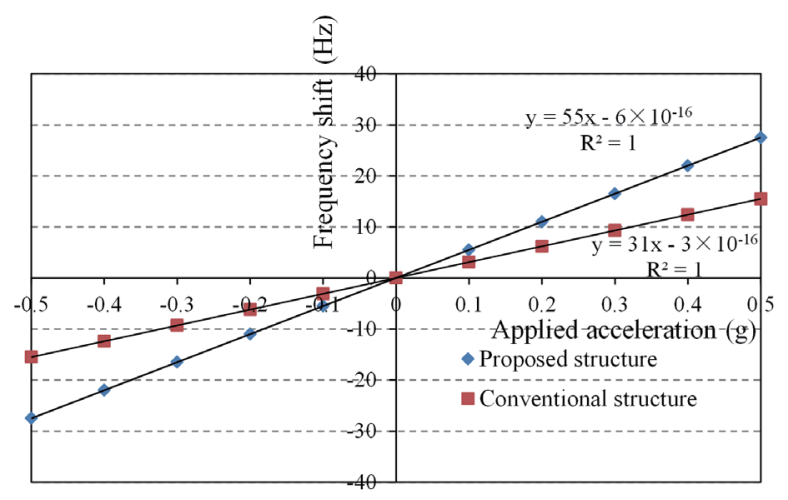

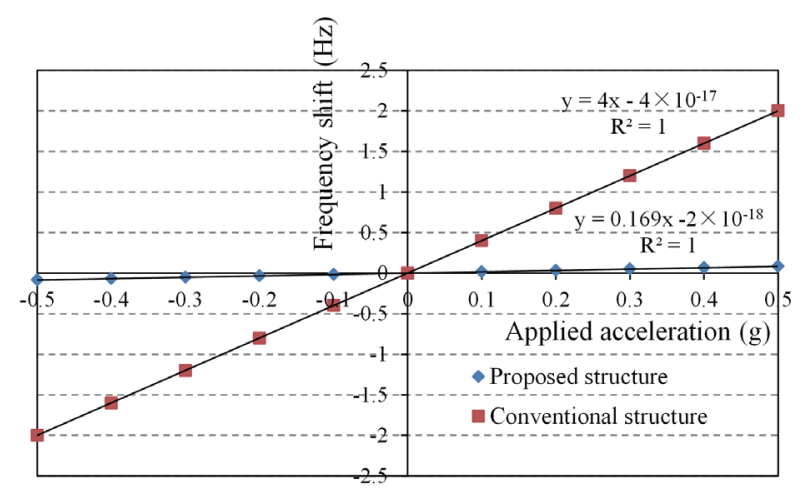

(b) (a)

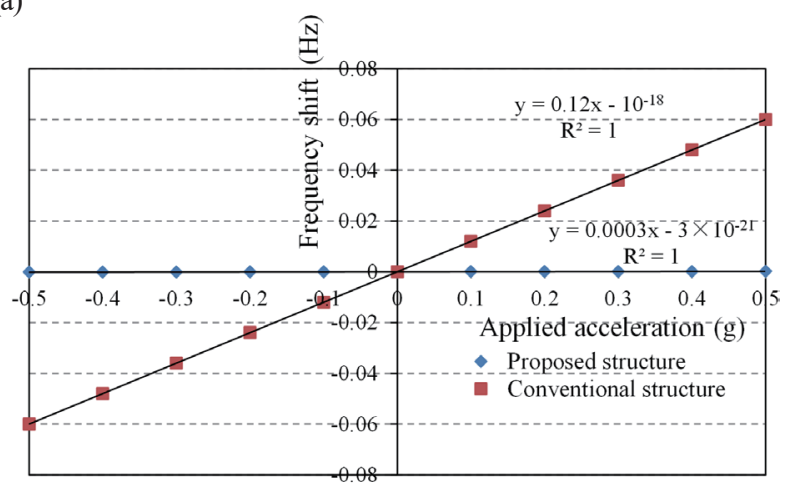

(c)

Fig. 11. (Color online) Simulated results of the proposed sensor and ONERA structure. (a) Measurement direction $\left(a_{1}\right)$, (b) $Y$ direction $\left(a_{2}\right)$, and (c) $X$ direction $\left(a_{3}\right)$.

There are several ways to further improve the performance of the accelerometer. The most direct way is to improve the vibration characteristics of the vibrating beam resonator. As shown in Fig. 6(b), the motional resistance is as large as $7.6 \mathrm{M} \Omega$ even after vacuum sealing, which makes it difficult to drive such a resonator. The large motional resistance is caused by the simple surface electrodes, as shown in Fig. 5(b), and only part of the length of the beam is covered with metal electrodes, lowering the electromechanical coupling efficiency. As the next step, we will try to arrange the excitation electrode pattern on the entire beam length by improving the fabrication process. Another way to improve the performance is to design a differential structure that can double the scale factor and eliminate common-mode errors. It is also expected that the temperature stability can be improved.

\section{Conclusions}

A novel monolithic QVBA with high uniaxial sensitivity was designed and fabricated. A two-step wet etching process was also successfully designed to fabricate the proposed sensor. The natural frequency of the sensor was $51.58 \mathrm{kHz}$ and the scale factor was $54.46 \mathrm{~Hz} / \mathrm{g}$. Furthermore, a high Q value of 8000 was achieved and the bias stability was evaluated to be 33 $\mu g(1 \sigma)$ over $1 \mathrm{~h}$. Excellent temperature frequency stability of $1.9 \mathrm{ppm} /{ }^{\circ} \mathrm{C}$ was observed in the 
temperature range of -40 to $60{ }^{\circ} \mathrm{C}$. These results demonstrate that the novel structure can be used as a high-precision inertial sensor.

\section{Acknowledgments}

This work was financially supported by the Foundation of Key Laboratory of Micro-Inertial Instrument and Advanced Navigation Technology, Ministry of Education, China (201401), the Natural Science Foundation of Jiangsu Province (BK20171366), and the Fundamental Research Funds for the Central Universities (2242016K40041, 2242017K1G009).

\section{References}

1 R. M. Langdon: J. Phys. E: Sci. Instrum. 18 (1985) 103.

2 J. E.-Y. Lee, B. Bahreyni, and A. A. Seshia: Sens. Actuators, A 148 (2008) 395.

3 L. Huang, H. Yang, Y. Gao, L. Zhao, and J. Liang: Sens. 13 (2013) 15785.

4 Y. Yin, Z. Fang, F. Han, B. Yan, J. Dong, and Q. Wu: Sens. Actuators, A 268 (2017) 52.

5 X. Zou, P. Thiruvenkatanathan, and A. A. Seshia: J. Microelectromech. Syst. 23 (2014) 768.

6 Y. Zhao, J. Zhao, X. Wang, G. Xia, A. Qiu, Y. Su, and Y. Xu: IEEE J. Solid-State Circuits 50 (2015) 2113.

7 E. D. Reedy and W. J. Kass: IEEE Trans. Ultrason. Ferroelectr. Freq. Control 37 (1990) 464.

8 C. Li, Y. Zhao, B. Li, R. Cheng, D. Sun, C. Han, and Y. Zhao: Sens. Actuators, A 253 (2017) 1.

9 J. Liang, L. Zhang, L. Wang, Y. Dong, and T. Ueda: Sensors 15 (2015) 22049.

10 S. W. Yoon, S. W. Lee, N. C Perkins, and K. Najafi: J. Micromech. Microeng. 21 (2011) 015017.

11 S. W. Yoo, S. W. Lee, N. C. Perkins, and K. Najafi: J. Microelectromech. Syst. 20 (2011) 1016.

12 O. L. Traon, D. Janiaud, S. Muller, and P. Bouniol: Proc. Position, Location and Navigation Symp. (1998) $25-37$.

13 O. L. Traon, D. Janiaud, and S. Muller: US Patent No. 5962786 (1999).

14 O. L. Traon, D. Janiaud, M. Pernice, S. Masson, S. Muller, and J.-Y. Tridera: Pro. 2006 IEEE PLANS, Position Location and Navigation Symp. (2006) 6-15.

15 T. Loret, G. Hardy, C. Vallée, V. Demutrecy, T. Kerrien, S. Cochain, D. Boutoille, R. Taïbi, and R. Blondeau: Proc. Inertial Sensors and Systems Symp. (ISS), 2014 DGON (2014) 1-14.

16 J. Liang, Y. Chai, G. Meng, and X. Chen: Micro Nano Lett. 8 (2013) 52.

17 R. Levy, D. Janiaud, J. Guerard, R. Taïbi, and O. Le Traon: Proc. 2014 Int. Symp. Inertial Sensors and Systems (2014) $1-4$.

18 M. Weinberg, R. Candler, S. Chandorkar, J. Varsanik, and T. Kenny, A. Duwel: Proc. 2009 Solid-State Sensors, Actuators and Microsystems Conf. (Transducers 2009) 688-695.

19 W. C. Albert: Proc. 1994 IEEE 48th Int. Frequency Control Symp. (IEEE, 1994) 415-420.

20 J. Liang, F. Kohsaka, T. Matsuo, and T. Ueda: IEEJ Trans. Sens. Micromachines 127 (2007) 337.

\section{About the Authors}

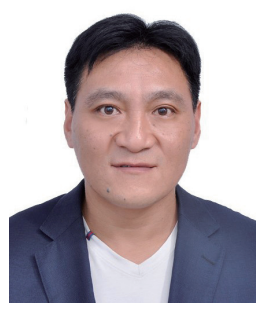

Jinxing Liang received his B.E. degree in mechanical engineering from China University of Mining and Technology, China, in 1998, his M.E. degree in electrical engineering from Kyushu University, Japan, in 2004, and his $\mathrm{Ph} . \mathrm{D}$. degree in measurement technology from Waseda University, Japan, in 2009. Since 2009, he has been an associate professor at the School of Instrument Science and Engineering in Southeast University. His research interests include quartz micromachining, quartz MEMS sensors, and microsensors. (j-liang@seu.edu.cn) 


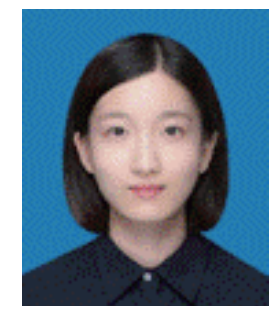

Qi Tang received her B.S. degree from Northwest Polytechnical University, China, in 2018. Since 2018, she has been a master's student at Southeast University. Her research interests include MEMS, magnetism, and sensors. (220183247@seu.edu.cn)

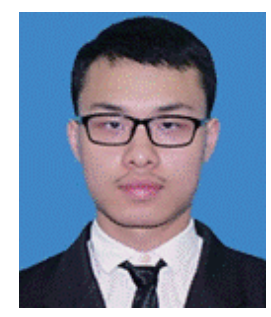

Shengshou Lin received his B.E. degree from Nanjing University of Science \& Technology, China, in 2020. Since 2020, he has been a master's student at Southeast University, China. His research interests are in MEMS, mechatronics, and sensors.(220203459@seu.edu.cn) 